\title{
Macro- and microvascular functions in cystic fibrosis adults without cardiovascular risk factors: A case-control study
}

\author{
Enrico Vizzardi ${ }^{*}$, Edoardo Sciatti ${ }^{*}$, Ivano Bonadei ${ }^{1}$, Dario S. Cani ${ }^{1}$, Elisa Menotti ${ }^{1}$, Francesco Prati ${ }^{1}$, \\ Lucia Dallapellegrina1, Marco Metra' ${ }^{1}$, Marialma Berlendis ${ }^{2}$, Piercarlo Poli³, Rita Padoan ${ }^{3}$ \\ ${ }^{1}$ Section of Cardiovascular Diseases, Department of Medical and Surgical Specialties, Radiological Sciences and \\ Public Health, University of Brescia; ${ }^{2}$ Pulmonology Unit, ASST Spedali Civili of Brescia; ${ }^{3}$ Cystic Fibrosis Center, \\ Pediatric Department, University and ASST Spedali Civili of Brescia, Italy; "These authors contributed equally
}

\begin{abstract}
Increasing survival from cystic fibrosis show untypical systems involvement, such as cardiocirculatory. In particular, the presence of CFTR in smooth muscle and endothelial cells, systemic inflammation and oxidative stress could explain vascular alterations in these patients. We aimed at noninvasely evaluating macro- and microvascular dysfunction in cystic fibrosis adults without cardiovascular risk factors. Twenty-twoadults affected by cystic fibrosis and 24 healthy volunteers matched for age and sex were enrolled. None had known cardiovascular risk factors. All people underwent blood pressure measurement, microvascular function assessment by EndoPAT-2000 device (calculating RH-PAT index) and macrovascular evaluation by pulse wave velocity (PWV). RHPAT index was significantly lower in patients than in controls $(1.74 \pm 0.59$ vs $2.33 \pm 0.34 ; \mathrm{p}<0.001)$. Thirteen patients of $22 \mathrm{had}$ a value inferior to the threshold of 1.67 (59.1\%), while no controls
\end{abstract}

Correspondence: Dr. Edoardo Sciatti, Department of Medical and Surgical Specialties, Radiological Sciences and Public Health, University of Brescia, Piazzale Spedali Civili 1, 25123 Brescia, Italy. Tel. +39.030.3995536 - Fax: +39.030.3995013.

E-mail: edoardo.sc@tin.it

Contributions: EV, ES, MB, RP, paper writing; ES, data analysis; ES, IB, DC, EM, FP, LD, PP, data collection; MB, RP, MM, paper revision.

Conflict of interest: The Authors declare no conflict of interest.

Acknowledgments: The Authors are grateful to "Lega Italiana Fibrosi Cistica - onlus Lombardia" for its interest in helping these patients.

Keywords: Cystic fibrosis; endothelial dysfunction; EndoPAT; pulse wave velocity; vascular explorer; vascular function.

Received for publication: 16 January 2019.

Accepted for publication: 6 May 2019.

(C) Copyright E. Vizzardi et al., 2019

Licensee PAGEPress, Italy

Monaldi Archives for Chest Disease 2019; 89:1035

doi: 10.4081/monaldi.2019.1035

This article is distributed under the terms of the Creative Commons Attribution Noncommercial License (by-nc 4.0) which permits any noncommercial use, distribution, and reproduction in any medium, provided the original author(s) and source are credited. had $(\mathrm{p}<0.001)$. Carotid-femoral PWV did not differ between the two groups $(5.2 \pm 1.5 \mathrm{~m} / \mathrm{s}$ vs $5.4 \pm 1.1 ; \mathrm{p}=0.9)$, while brachial-ankle one did $(11.0 \pm 2.2 \mathrm{~m} / \mathrm{s}$ vs $10.1 \pm 0.8 \mathrm{~m} / \mathrm{s} ; \mathrm{p}=0.04)$. Adults patients affected by cystic fibrosis show peripheral endothelial dysfunction, which is the first alteration in atherosclerotic phenomenon. Moreover, arterial stiffness measured by PWV unclearly seems to differ respect of healthy people, perhaps because PWV alterations are typical of above 50 years old people. It is unclear what prognostic role of future developing of atherosclerotic disease these findings could be, but it seems evident that cystic fibrosis directly affects cardiovascular system itself.

\section{Introduction}

Cystic fibrosis (CF) is the most common life-threatening autosomic recessive disease in Caucasian race, involving approximately $1 / 2500$ newborns. It is caused by more than 1900 different mutations of CF transmembrane conductance regulator (CFTR) gene, located on the $7^{\text {th }}$ chromosome and codifying for a transmembrane chlorine channel. Typical symptoms regard the pneumological and gastrointestinal systems. Despite of the comorbidities and the severity of this pathology, survival is still increasing; in fact today about $50 \%$ of patients join adulthood $[1,2]$. A prolonged survival, thus, favors the appearance of new complications and the involvement of other systems [3]. In particular, the presence of chronic inflammation due to the pathophysiology of the transmembrane channel in the airways leads to particular consequences in heart and blood vessels [4]. Arterial stiffening is a hallmark of the ageing process in healthy people, in atherosclerotic patients and even in those who suffer from chronic obstructive pulmonary disease [5-8]. Moreover, it predicts cardiovascular (CV) adverse events, such as myocardial infarction, heart failure and overall mortality [9]. Few studies have been published to explore vascular changes in CF but their prognostic implications are still unknown. The aim of the present study was to explore both macrovascular (arterial stiffness) and microvascular (peripheral endothelium) functions in adult patients affected by $\mathrm{CF}$, compared with healthy controls.

\section{Patients and Methods}

We enrolled 22 patients affected by CF, followed by the Cystic Fibrosis Center, Pediatric Department, University of Brescia and 
the Pulmonology Unit of ASST Spedali Civili of Brescia, Italy. They were compared with 24 healthy volunteers matched for age, sex and body mass index (BMI). Cases and controls were accurately selected without known CV risk factors. Every one underwent blood pressure measurement, micro- and macrovascular evaluation.

\section{Pressure measurement}

Blood pressure was assessed using a standard, calibrated sphygmomanometer. The mean of three sitting and standing blood pressure was recorded. The arm in which the highest sitting diastolic pressures was found was the arm used for all subsequent readings throughout the study. Every effort was made to have the same staff member obtain blood pressure measurements in each individual patient, at the same time of day, using the same equipment. Systolic pressure was recorded when the initial sound is heard (Phase I of the Korotkoff sound), while diastolic pressure at the disappearance of the sound (Phase V of the Korotkoff sound). The cuff was deflated at a rate not greater than $2 \mathrm{mmHg} / \mathrm{sec}$.

\section{Spirometrical test}

Lung function was measured evaluating: Slow vital capacity (SVC), inspiratory capacity (IC), flow-volume curve with evaluation of forced expiratory volume (FEV1), forced vital capacity (FVC), Tiffeneau index (FEV1/SVC), mean forced expiratory flow (FEF25-75\%), plethysmographic pulmonary volumes with calculus of total lung capacity (TLC), functional residual capacity (FRC), residual volume (RV), carbon monoxide diffusing capacity (DLCO), respiratory muscle strength (maximal inspiratory and expiratory pressure: MIP and MEP).

Pulmonary function tests were obtained using Vmax 22 pulmonary function analysis machines: spirometer with mass flow meter and Autobox 6200 (Sensormedics, Yorba Linda, CA, USA). The American Thoracic Society guidelines were used to perform the tests of pulmonary function. The theoretical values used as reference were those reported by ATS/ERS [10] and Zapletal [11].

\section{Peripheral endothelial function}

Microvascular function was measured with peripheral arterial tonometry (PAT) signals, obtained using the EndoPAT-2000 device (Itamar Medical Ltd., Caesarea, Israel), which has been validated and used previously to assess peripheral arterial tone in other populations [12-16]. Specially designed finger probes were placed on the middle finger of each subject's hands. These probes comprised a system of inflatable latex air cuffs connected by pneumatic tubes to an inflating device controlled through a computer algorithm. A constant counter pressure (pre-determined by baseline diastolic blood pressure) was applied through the air cushions. This prevented venous pooling thus avoiding venoarteriolar reflex vasoconstriction. There was no occlusion of arterial blood flow. Pulsatile volume changes of the distal digit induced pressure alterations in the finger cuff, which were sensed by pressure transducers and transmitted to and recorded by the EndoPAT-2000 device. A decrease in the arterial blood volume in the distal fingertip caused a decrease in pulsatile arterial column changes, reflected as a decrease in the measured PAT signal, and vice versa. Endothelial function was measured via reactive hyperemia-PAT index (RH-PAT index): a reactive hyperemia protocol consisted of a 5 min baseline measurement, after which a blood pressure cuff on the test arm was inflated to $60 \mathrm{mmHg}$ above baseline systolic blood pressure or at least $200 \mathrm{mmHg}$ for $5 \mathrm{~min}$. Occlusion of pulsatile arterial flow was confirmed by the reduction of the PAT tracing to zero. After $5 \mathrm{~min}$, the cuff was deflated, and the PAT tracing was recorded for a further $5 \mathrm{~min}$. The ratio of the PAT signal after cuff release compared with baseline was calculated through a computer algorithm automatically normalizing for baseline signal and indexed to the contra lateral arm. The calculated ratio normal value was above 1.67 , while $\leq 1.67$ was considered endothelial dysfunction.

\section{Pulse wave analysis}

To evaluate vascular rigidity we used applanation tonometry with Vascular Explorer (Enverdis GmbH, Jena, Germany); it calculated arterial stiffness parameters from oscillatory recorded pressure waves of the brachial and anterior tibial arteries. Using inflatable upper and lower arm cuffs with high fidelity sensors, pulsatile volume changes (resulting from pulsatile fluctuations of the arteries) were transduced into pressure curves. Pulse waves were recorded when the arteries were completely occluded at a cuff pressure that was $35-40 \mathrm{mmHg}$ above systolic blood pressure. A computer software was used to further analyze the recorded pulse waves. Pulse transit time (PTT) was determined from the decomposition of the general aortic pressure wave using the reflection method. This measurement is based on the fact that the forward traveling pulse wave (generated by the ejection of the left ventricle) is reflected in the periphery creating a second reflected wave. PTT was determined from the difference in milliseconds between the forward and the beginning of the reflected pressure wave, and aortic pulse wave velocity (PWV) was automatically calculated from PTT and travelling distance between jugulum (sternal notch) and symphysis pubica (according to manufacture recommendations). We evaluated three different PWVs calculated by the software: brachial-ankle PWV was registered by means of simultaneous cuff measurements taken on the upper arm and ankle at diastolic pressure (foot-foot measurement of the time difference between both pressure waves), aortic PWV was measured using the reflection method under brachial stop/flow conditions (footfoot measurement of the time difference for the direct pulse wave and the pulse wave reflected at the bifurcation), while carotidfemoral PWV was calculated from brachial-ankle and aortic PWVs. We followed the European recommendations for carotidfemoral PWV, multiplying for 0.8 the ratio between the measured distance between jugulum and symphysis pubica and the PTT [17]. Brachial-ankle PWV was calculated using the following equation: $(\mathrm{La}-\mathrm{Lb}) / \Delta \mathrm{Tba}(\mathrm{cm} / \mathrm{s})$, while $\Delta \mathrm{Tb}$ a was the PTT between brachium and ankle, $\mathrm{Lb}$ and $\mathrm{La}$ the path lengths from the suprasternal notch to the brachium $(\mathrm{Lb})$ and to the ankle $(\mathrm{La})$, calculated using following equations: $\mathrm{Lb}=0.2195^{*}$ height $(\mathrm{cm})-2.0734, \mathrm{La}=$ $0.8129 *$ height $(\mathrm{cm})+12.328[18,19]$.

\section{Statistical analysis}

All analyses were done using IBM SPSS Statistics 20 for Windows (SPSS, Inc., Chicago, IL, USA). Continuous variables were visually tested for normality by Q-Q plots and represented by mean \pm standard deviation or median (interquartile range), while categorical variables as frequency (n) and percentage of the sample. Independent samples Student's t test or Mann-Whitney U test were performed to analyze the difference between means for continuous variables and Fisher's exact test for the difference between proportions for dichotomic ones. Spearman's bivariate correlation was run between every vascular parameter and variables regarding pulmonary involvement (spirometrical data and microbiological colonization) and inflammation (C-reactive protein). For all statistical tests, probability values $<0.05$ were considered significant. 


\section{Results}

Demographic and clinical characteristics are shown in Table 1. Patients and controls did not differ in age, BMI and sex distribution. Fifteen patients out of 22 were males $(68.2 \%)$ while 17 healthy volunteers out of 24 were $(70.8 \% ; p=1)$. No patients were suffering from $\mathrm{CF}$ had secondary diabetes mellitus, nor any other $\mathrm{CV}$ risk factor. No patients were on CFTR potentiators or correctors. Creactive protein was increased in the patients' group (median 95 $\mathrm{ng} / \mathrm{L}$, interquartile range $16-243 \mathrm{ng} / \mathrm{L}$ ).

All people in the study population were normotensive. Systolic blood pressure was not statistically different between the two groups, while diastolic blood pressure was slightly higher among patients than controls, as well as heart rate. On the contrary, pulse pressure was similar between the groups. Spirometrical data of the cases are displayed in Table 2 as percentage of theoretical values. They are typical of pulmonary obstructive diseases. In particular,
14 patients $(63.6 \%)$ showed a FEF25-75\% value $<60 \%$ and 13 $(59.1 \%)$ a FEV $1<80 \%$. Of them, 3 patients $(13.6 \%)$ had a value $<$ $40 \%, 4(18.2 \%)$ between 40 and $60 \%$, and $6(27.3 \%)$ between 60 and $80 \%$. Regarding chronic airways microbiological colonization, in 11 patients $(50.0 \%)$ Pseudomonas aeruginosa was found, in 3 (13.6\%) methicillin-resistant Staphylococcus aureus, and in other 5 $(22.7 \%)$ both bacteria. Microvascular and macrovascular functions data are resumed in Table 3. Microvascular function expressed as RH-PAT index was significantly lower in patients than in controls. Indeed, 13 patients out of 22 had a value below the threshold of 1.67 (59.1\%), while no controls had $(\mathrm{p}<0.001)$.

Macrovascular function measured as PWV did not differ between the two groups regarding carotid-femoral one, while brachial-ankle one was slightly higher for cases.

Patients with chronic airways microbiological colonization had similar RH-PAT index ( $1.78 \pm 0.62$ vs $1.51 \pm 0.33, \mathrm{p}=0.586)$, carotidfemoral PWV $(5.2 \pm 1.5$ vs $5.0 \pm 1.9, \mathrm{p}=0.718)$ and brachial-ankle PVW (11.2 \pm 1.5 vs $9.7 \pm 5.2, \mathrm{p}=0.464)$ than the others. Endothelial

Table 1. Demographic and clinical characteristics of the study population.

\begin{tabular}{lccc} 
Variable & Cystic fibrosis $(\mathrm{n}=22)$ & Healthy controls $(\mathrm{n}=24)$ & p-value \\
Age (years) & $24(21-30)$ & $32(23-37)$ & 0.3 \\
Male sex & $15(68.2 \%)$ & $17(70.8 \%)$ & 1 \\
\hline BMI $(\mathrm{kg} / \mathrm{m} 2)$ & $22.0(19.3-23.9)$ & $22.2(21.0-24.2)$ & 0.7 \\
BSA (m2) & $1.75(1.56-1.89)$ & $1.75(1.68-1.88)$ & 0.6 \\
SBP (mmHg) & $120(111-130)$ & $120(110-130)$ & 0.5 \\
DBP (mmHg) & $80(70-80)$ & $70(60-75)$ & 0.01 \\
PP (mmHg) & $50(38-54)$ & $50(43-59)$ & 0.3 \\
HR (bpm) & $81(70-90)$ & $68(64-78)$ & 0.01 \\
\hline
\end{tabular}

BMI, body mass index; BSA, body surface area; SBP, systolic blood pressure; DBP, diastolic blood pressure; PP, pulse pressure; HR, heart rate.

Table 2. Spirometrical data of the cystic fibrosis patients. Data expressed as \% of theoretical values; mean \pm standard deviation (minimum-maximum).

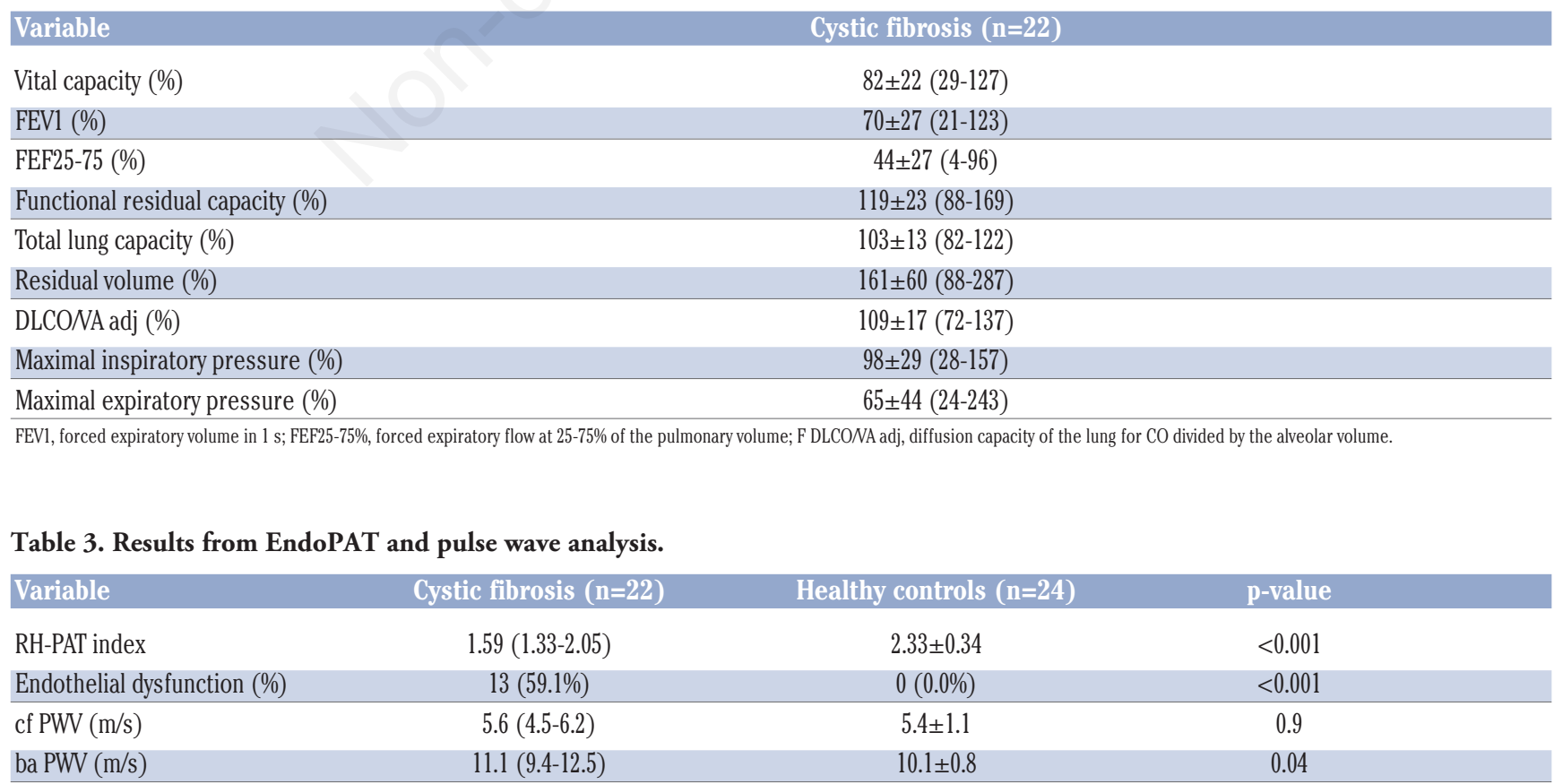

RH-PAT, reactive hyperemia-peripheral arterial tonometry index; cf, carotid-femoral; PWV, pulse wave velocity; ba, brachial-ankle. 
dysfunction was similarly present in both groups $(57.9 \%$ vs $66.7 \%$, $\mathrm{p}=0.642$ ). Interestingly, among all tested correlations, RH-PAT index and FEV1 showed a significant association with C-reactive protein $(\rho=-0.482, p=0.023$ and $\rho=-0.452, p=0.035$, respectively).

\section{Discussion}

For the first time to date, to our knowledge, peripheral endothelial function measured by EndoPAT-2000 device was evaluated in people affected by CF. This method was demonstrated to be operator-independent and highly useful in people without $\mathrm{CV}$ diseases. Endothelial dysfunction is considered the first vascular alteration occurring in the atherosclerotic phenomenon and several $\mathrm{CV}$ diseases. Its evaluation is a good way to analyze one's CV health [12-16]. Our data suggest that adult patients suffering from CF and without CV risk factors have a higher frequency of endothelial dysfunction compared with healthy people. In particular, the decrease of endothelial function is associated with inflammation and FEV1. However, to our knowledge, only two studies investigated the presence of endothelial dysfunction in vivo in these patients, using strain-gauge venous-occlusion plethysmography [20] and brachial flow mediated dilation [21]. Therefore, our study confirms that of McGrath et al. [20] and that of Poore et al. [21], but using a different method. In fact, some Authors reported that flow mediated dilation and EndoPAT represent two different point of view on peripheral endothelial function: on elastic artery the first, on microcirculation the second [22]. Recently microvascular dysfunction in CF patients has been confirmed using laser-doppler imaging through postocclusive reactive hyperemia, local thermal hyperthermia and iontophoresis with acetylcholine, compared with age matched control patients [23]. CFTR is present in smooth muscle cells [24,25] and in vascular endothelium [26]. Its alteration can reduce nitric oxide (NO) bioavailability (controlling the phosphorylation and activity of endothelial NO synthase) and decrease endothelial function. CF has shown to be poorly related to traditional CV risk factors, whilst strongly associated with other biomarkers of premature vascular ageing as activated inflammatory-immune processes and oxidative stress [27]. Moreover, CF is characterized by inflammatory state and oxidative stress $[28,29]$, two conditions that reduce NO bioavailability [30]. Systemic inflammation could also influence endothelial function, which is related to arterial stiffness and a predictor of global CV risk [31]. Additionally, CFTR dysfunction may disrupt endothelial barrier function favoring leukocyte infiltration and inflammation. Especially under shear stress the impact of the CFTR dysfunction is evident on the stability and morphology of endothelial cells. Furthermore, the release of microvesicles from endothelial cells of CF patients does not seem to stimulate cell proliferation sufficiently [32]. Besides that, the CFTR block stimulates the production of IL-8 powerful chemotactic agent. Summarizing, on the one hand, a reduced endothelial integrity, on the other, a greater attraction and infiltration of leukocytes may play a role in determining endothelial dysfunction in these patients. What is the real cause of endothelial dysfunction in CF is still unknown and specific studies are needed, but we found a correlation between RH-PAT index and C-reactive protein.

Regarding macrovascular function, in our study carotid-femoral PWV did not differ between patients and controls, while brachialankle PWV did. These results partly confirm those of Hull et al. [33], who demonstrated that systemic inflammation in these patients determines an increase in augmentation index (AIx) and so a predisposition to heart diseases in survived adults [33]. Vice versa, in their paper PWV was not different between patients and controls [33]. In addition, AIx was associated to C-reactive protein, underlying the role of systemic inflammation in vascular changes $[33,34]$. Buehler et al. demonstrated increased stiffness in large arteries in CF children, especially if colonized by Pseudomonas aeruginosa or Stenotrophomonas maltophilia. According to the authors, the increase in arterial stiffness seems to be related to systemic inflammation [35]. In fact, there is a curve-linear relationship between age and the aortic PWV; thus, age-related changes in aortic PWV are less marked in younger subjects and increase after 50 years of age [36]. Unfortunately, our study lacks the evaluation of AIx, which greater changes in younger individuals and was shown different between patients and controls by Hull et al. [33]. We hypothesize that a sort of vascular remodeling is present in these patients but both Hull and colleagues' and our study are underpowered to catch it.

Strength of our study is the careful selection of patients without $\mathrm{CV}$ risk factors nor overt pulmonary hypertension. In this way we have been able to identify vascular alterations linked to CF itself. Nevertheless, our cohort was characterized by a great damage in expiratory flows at low level of pulmonary filling, as expressed by FEF25-75\%, meaning peripheral airways largely involve in lung remodeling. Minimum value is $4 \%$ of theoretical one and indicates a severe lung damage. Moreover, FEV1 is the "gold standard" parameter in the follow-up and in the prognosis estimation of CF patients. It represents the patency to airflow of the larger airways. Again, minimum value is $21 \%$ and suggests a severe impairment. In the present study we compared two groups of adults without known CV risk factors; the only difference between them was the presence of CF.

Our study suffers from some limitations. First, the small number of patients in the cohort, which prevent us from finding important correlations regarding, for example, microbiological colonization and vascular parameters. Second, we lack a direct correlation between vascular parameters and disease outcome.

Insofar, our study shows the presence of endothelial dysfunction in adult patients suffering from $\mathrm{CF}$ without $\mathrm{CV}$ risk factors. On the other hand, it is still unclear whether arterial stiffness is largely present in such disease. Moreover, we do not know what prognostic role of future developing of atherosclerotic disease these findings could be, but it seems evident that CF directly affects CV system itself. Future studies are needed to explore CV outcomes of these patients in order to identify those who may benefit from cardiologic primary prevention therapies.

\section{References}

1. Cystic Fibrosis Foundation Patient Registry. Annual Data Report. Bethesda: Cystic Fibrosis Foundation; 2017.

2. Hurley MN, McKeever TM, Prayle AP, et al. Rate of improvement of CF life expectancy exceeds that of general population-observational death registration study. J Cyst Fibros 2014; 13:410-5.

3. Ionescu AA, Nixon LS, Evans WD, et al. Bone density, body composition and inflammatory status in cystic fibrosis. Am J Respir Crit Care Med 2002;162:789-94.

4. MacNee W, Maclay J, McAllister D. Cardiovascular injury and repair in chronic obstructive pulmonary disease. Proc Am Thorac Soc 2008;5:824-33.

5. Vaitkevicius P, Fleg J, Ingel J, et al. Effects of age and the aerobic capacity on arterial stiffness in health adults. Circulation 1993;88:1456-62. 
6. Zieman SJ, Melenovsky V, Kass DA. Mechanisms, pathophysiology and therapy of arterial stiffness. Arterioscler Thromb Vasc Biol 2005;25:932-43.

7. Maclay JD, McAllister DA, Mills NL, et al. Vascular dysfunction in chronic obstructive pulmonary disease. Am J Respir Crit Care Med 2009;180:513-520.

8. Hamilton PK, Lockhart CJ, Quinn CE, et al. Arterial stiffness: clinical relevance, measurement and treatment. Clin Sci 2007;113:157-70.

9. Safar ME, Levy BI, Struijker-Boutier H. Current perspectives on arterial stiffness and pulse pressure in hypertension and cardiovascular diseases. Circulation 2003;107:2864-9.

10. Pellegrino R, Viegi G, Brusasco V, et al. Interpretative strategies for lung function tests. Eur Respir J 2005;26:948-68.

11. Zapletal A, Paul T, Samánek M. [Significance of contemporary methods of lung function testing for the detection of airway obstruction in children and adolescents (author's transl)]. Z Erkr Atmungsorgane 1977;149:343-71.

12. Goor DA, Sheffy J, Schnall RP et al. Peripheral arterial tonometry: a diagnostic method for detection of myocardial ischemia induced during mental stress tests: a pilot study. Clin Cardiol 2004;27:137-41.

13. Bonetti PO, Barsness GW, Keelan PC, et al. Enhanced external counterpulsation improves endothelial function in patients with symptomatic coronary artery disease. J Am Coll Cardiol 2003;41:1761-8.

14. Bonetti PO, Lerman LO, Lerman A. Endothelial dysfunction: a marker of atherosclerotic risk. Arterioscler Thromb Vasc Biol 2003;23:168-75.

15. Lavie P, Shlitner A, Sheffy J, Schnall RP. Peripheral arterial tonometry: a novel and sensitive non-invasive monitor of brief arousals during sleep. Isr Med Assoc J 2000 2:246-47.

16. Halligan SC, Murtagh B, Lennon RJ, et al. Effect of long-term hormone replacement therapy on coronary endothelial function in postmenopausal women. Mayo Clin Proc 2004;79:1514-20.

17. Reference Values for Arterial Stiffness' Collaboration. Determinants of pulse wave velocity in healthy people and in the presence of cardiovascular risk factors: 'establishing normal and reference values'. Eur Heart J 2010;31:2338-50.

18. Munakata M, Ito N, Nunokawa T, Yoshinaga K. Utility of automated brachial ankle pulse wave velocity measurements in hypertensive patients. Am J Hypertens 2003;16:65-7.

19. Yamashina A, Tomiyama $H$, Takeda K, et al. Validity, reproducibility, and clinical significance of noninvasive brachial-ankle pulse wave velocity measurement. Hypertens Res 2002;25:359-64.

20. McGrath LT, McCall D, Hanratty CG, et al. Individuals with cystic fibrosis do not display impaired endothelial function or evidence of oxidative damage in endothelial cells exposed to serum. Clin Sci (Lond) 2001;101:507-13.

21. Poore S, Berry B, Eidson D, McKie KT, Harris RA. Evidence of vascular endothelial dysfunction in young patients with cystic fibrosis. Chest 2013;143:939-45.

22. Flammer AJ, Anderson T, Celermajer DS, et al. The assessment of endothelial function: from research into clinical practice. Circulation 2012;126:753-67.

23. Rodriguez-Miguelez P, Thomas J, Seigler N, et al. Evidence of microvascular dysfunction in patients with cystic fibrosis. Am J Physiol Heart Circ Physiol 2016;310:H1479-85.

24. Robert R, Thoreau V, Norez C, et al. Regulation of the cystic fibrosis transmembrane conductance regulator channel by betaadrenergic agonists and vasoactive intestinal peptide in rat smooth muscle cells and its role in vasorelaxation. J Biol Chem 2004;279:21160-8.

25. Michoud MC, Robert R, Hassan M, et al. Role of the cystic fibrosis transmembrane conductance channel in human airway smooth muscle. Am J Respir Cell Mol Biol 2009;40:217-22.

26. Tousson A, Van Tine BA, Naren AP, et al.. Characterization of CFTR expression and chloride channel activity in human endothelia. Am J Physiol 1998;275:C1555-64.

27. Reverri EJ, Morrisey BM, Cross CE, Steinberg FM. Inflammation, oxidative stress, and cardiovascular disease risk factors in adults with cystic fibrosis. Free Radic Biol Med 2014;76:261-77.

28. Wood LG, Fitzgerald DA, Gibson PG, et al. Oxidative stress in cystic fibrosis: dietary and metabolic factors. J Am Coll Nutr 2001;20:S157-65.

29. Elizur A, Cannon CL, Ferkol TW. Airway inflammation in cystic fibrosis. Chest 2008;133:489-95.

30. Rifai N, Ridker PM. Inflammatory markers and coronary heart disease. Curr Opin Lipidol 2002;13:383-9.

31. Halcox JP, Schenke WH, Zalos G, et al. Prognostic value of coronary vascular endothelial dysfunction. Circulation 2002 6;106:653-8.

32. Totani L, Plebani R, Piccoli A, et al. Mechanisms of endothelial cell dysfunction in cystic fibrosis. Biochim Biophys Acta Mol Basis Dis 2017; 1863:3243-53.

33. Hull JH, Garrod R, Ho TB, et al. Increased augmentation index in patients with cystic fibrosis. Eur Respir J 2009;34;1322-8.

34. Yasmin, McEniery CM, Wallace S, et al. C-reactive protein is associated with arterial stiffness in apparently healthy individuals. Arteriosceler Thromb Vasc Biol 2004;24:969-74.

35. Buehler T, Steinmann M, Singer F, et al. Increased arterial stiffness in children with CF. Eur Resp J 2012;39:1536-7.

36. McEniery CM, Wilkinson IB, Avolio AP. Age, hypertension and arterial function. Clin Exp Pharmacol Physiol 2007;34:665-71. 
